\title{
Altered DNA methylation is associated with docetaxel resistance in human breast cancer cells
}

\author{
LENA KASTL, IAIN BROWN and ANDREW C. SCHOFIELD \\ School of Medicine and Dentistry, College of Life Sciences and Medicine, University of Aberdeen, \\ Medical School, Foresterhill, Aberdeen, AB25 2ZD, UK
}

Received October 20, 2009; Accepted November 30, 2009

DOI: 10.3892/ijo_00000607

\begin{abstract}
Docetaxel is an effective chemotherapy drug to treat breast cancer but the underlying molecular mechanisms of drug resistance are not fully understood. DNA methylation is an epigenetic event, involved in the control of gene expression, which is known to play an important role in cancer and chemotherapy drug resistance. To investigate the role of DNA methylation in docetaxel resistance in breast cancer we used two human breast cancer cell lines (MCF-7 and MDA-MB231) that were made resistant to docetaxel. Docetaxel-resistant sub-lines were treated with different concentrations of decitabine. Global methylation and DNA methyltransferase (DNMT) activity was measured using an ELISA-based assay. Quantitative real-time PCR was used to study DNMT gene expression. Cell viability was studied by MTT assay. Global methylation was increased in MCF-7 but not significantly changed in MDA-MB-231 docetaxel-resistant cells. Decreased DNMT activity and decreased DNMT1 and $D N M T 3 b$ mRNA expression was associated with docetaxel resistance in both cell lines. To investigate how the components of the DNA methylation machinery may contribute towards docetaxel resistance, decitabine (5-aza-2'deoxycytidine), an inhibitor of DNA methylation, was used. Decitabine treatment decreased global methylation, DNMT activity and DNMT1, DNMT3 $a$ and DNMT3b mRNA expression in MDA-MB-231 docetaxel-resistant cells. In contrast, decitabine-treated MCF-7 docetaxel-resistant cells showed increased DNMT1, DNMT3a and DNMT3b mRNA expression indicating a cell line specific effect of decitabine. Decitabine treatment increased resistance in MCF-7 docetaxel-resistant cells and in the parental MCF-7 and MDA-MB231 docetaxel-sensitive cell lines, however, it did not alter response to docetaxel in MDA-MB-231 docetaxelresistant cells. This study demonstrates that changes in the
\end{abstract}

Correspondence to: Dr Andrew C. Schofield, School of Medicine and Dentistry, College of Life Sciences and Medicine, University of Aberdeen, Medical School, Foresterhill, Aberdeen, AB25 2ZD, UK

E-mail: a.schofield@abdn.ac.uk

Key words: breast cancer, chemotherapy, DNA methylation, drug resistance
DNA methylation machinery are associated with resistance to docetaxel in breast cancer cells. The use of epigenetic therapies, as a strategy to overcome drug resistance, needs to be investigated more fully to determine their effectiveness in different cancers and for different chemotherapy drugs.

\section{Introduction}

Breast cancer is one of the most common cancer types in women with more than 44,000 incidences in the UK per year. Death rates are continuously on the decline due to improved awareness, screening and treatment options, including hormonal and targeted therapy, as well as chemotherapy. One of the most effective chemotherapy drugs used in the treatment of breast cancer is docetaxel (Taxotere ${ }^{\circledR}$ ), a taxane which is derived from the needles of the European yew tree. It inhibits depolymerisation of ß-tubulin and leads to apoptotic arrest in cancer cells. Various clinical trials have demonstrated docetaxel to be more effective than other chemotherapy drugs when used in the neoadjuvant setting $(1,2)$. The overall response to docetaxel therapy in patients developing recurrence is about $50 \%$ (2), however, many patients are either intrinsically resistant or acquire resistance during the course of chemotherapy treatment.

Several molecular mechanisms of docetaxel resistance have been identified although the precise mechanisms of drug resistance are poorly understood. The cellular target of docetaxel, ß-tubulin, which is the basic structural unit of microtubules and therefore important for structural integrity in cells, shows differential expression of $\beta$-tubulin isotypes in docetaxel-resistant breast cancer cells (3). Apoptotic pathways are also involved in docetaxel resistance, and antiapoptotic proteins such as bcl-2, if overexpressed, can prevent apoptosis induced by docetaxel (4). A third known mechanism involved in docetaxel resistance is overexpression of CYP3A4, which is the predominant enzyme that metabolizes docetaxel in the liver. Increased expression of $C Y P 3 A 4$ mRNA was present in breast tumours from patients who tended not to respond to docetaxel (5). Also, overexpression of the energy-dependent drug efflux pump P-glycoprotein can cause docetaxel resistance by accelerating drug efflux so that docetaxel is not accumulated properly within the cells and loses its effect (6).

There is evidence to suggest that epigenetic modifications such as histone de-acetylation and DNA methylation may act as potential causes of drug resistance $(7,8)$. Epigenetics can be described as a change in gene expression that is due to 
silencing of DNA, rather than mutations. DNA methylation occurs via three DNA methyltransferases (DNMT): DNMT1, DNMT3a, DNMT3b, which all add a methyl group to the C5 position of the cytosine ring of the DNA leading to 5-methylcytosine. This process can be reversed using DNA methylation inhibitors such as the widely studied, and used, inhibitor decitabine (5-aza-2'-deoxycytidine). It acts by binding to, and trapping, DNMTs and has recently been approved by the Food and Drug Administration to treat myelodysplastic syndrome. More interestingly, combination treatment with decitabine re-sensitised various cancer cells to chemotherapeutic drugs. Decitabine treatment was shown to reverse cisplatin resistance, which is common in ovarian cancer (9), as well as hypomethylating apoptotic genes therefore increasing response to doxorubicin in melanoma cells (10). In combination with paclitaxel, another taxane, inhibition of DNA methylation was associated with increased sensitivity in renal cell carcinoma (11). No study, however, has examined the effect of decitabine on docetaxel resistance in breast cancer. This study investigated the involvement of DNA methylation in two human breast cancer cell line models resistant to docetaxel. In addition, the effect of decitabine on response to docetaxel was studied in these cell lines to test the hypothesis that drug resistance may be overcome using a DNA methylation inhibitor.

\section{Materials and methods}

Cell culture. Human breast cancer cell lines (MCF-7 and MDA-MB-231) were obtained from the European Collection of Animal Cell Culture (Centre for Applied Microbiology and Research, Salisbury, UK). Each parental cell line (termed docetaxel-sensitive cells) was made resistant to docetaxel (termed docetaxel-resistant cells) following sequential exposure to docetaxel (Sanofi-Aventis, Surrey, UK) at increasing concentrations, as described previously (12). Cells were cultured and maintained in RPMI-1640 medium including L-glutamine, supplemented with $10 \%(\mathrm{v} / \mathrm{v})$ fetal calf serum and $1 \%(\mathrm{v} / \mathrm{v})$ penicillin/streptomycin $(100,000 \mathrm{U} / 1$ penicillin, $100 \mathrm{mg} / 1 \mathrm{streptomycin})$, at $37^{\circ} \mathrm{C}$ in a humidified atmosphere containing $5 \%$ carbon dioxide.

Drug treatment. Docetaxel solution was stored and prepared as recommended by the supplier. Fresh stock solutions of docetaxel were used for each experiment to prepare working dilutions. Cells were exposed to varying concentrations of 5-aza-2'-deoxycytidine (decitabine) (Sigma-Aldrich, Poole, UK), including $0.5,1,2,4$ and $8 \mu \mathrm{M}$, for $24 \mathrm{~h}$. Stock solutions of $500 \mu \mathrm{M}$ decitabine were prepared using cell growth media as described previously, and stored at $-80^{\circ} \mathrm{C}$. Fresh stock solutions of decitabine were prepared every 3-4 months, and fresh aliquots were used for each experiment. Untreated control cells consisted of cells and cell growth media only.

RNA extraction. RNA was extracted from TRIzol ${ }^{\circledR}$ reagent frozen cells, according to the manufacturer's protocol (Invitrogen, Paisley, UK). Cells were grown to $80 \%$ confluency and $1 \mathrm{ml}$ TRIzol reagent was added per flask and then further processed according to the protocol. RNA quality $\left(\mathrm{A}_{260} / \mathrm{A}_{280}\right.$ ratio) and quantity was determined by Nanodrop spectrophotometry (Nanodrop 1000 Spectrophotometer, Thermo Scientific, Loughborough, UK). RNA (2 $\mu \mathrm{g})$ was reverse transcribed with SuperScript ${ }^{\mathrm{TM}}$ II Reverse Transcriptase (Invitrogen), according to the manufacturer's protocol, using random hexamers (GE Healthcare, Buckinghamshire, UK).

Cell viability assay. In order to measure cell viability after treatment with decitabine, the MTT (3-(4,5-dimethylthiazol2-yl)-2,5-diphenyltetrazolium bromide) assay was used as described previously (12). Briefly, $5 \times 10^{4}$ cells were seeded onto a 96-well plate in $100 \mu \mathrm{l}$ medium and incubated for $24 \mathrm{~h}$ prior to docetaxel treatment. Appropriate docetaxel concentrations were added at $100 \mu 1(40 \mathrm{mg} / \mathrm{ml})$. MTT $(12 \mathrm{mM})$ (Sigma-Aldrich) was added to each well and cell viability was measured as described previously (12). For $24 \mathrm{~h}$ decitabine treatment, $50 \mu 1$ of the appropriate decitabine concentration $(0.5,1,2,4,8 \mu \mathrm{M})$ was added concurrently with docetaxel to each well.

Quantitative real-time PCR. Quantitative real-time PCR was performed using SYBR green master mix (Roche Diagnostics $\mathrm{GmbH}$, Mannheim, Germany), according to the following PCR conditions: initial denaturation at $95^{\circ} \mathrm{C}$ for $5 \mathrm{~min}$ followed by 45 cycles of amplification at $95^{\circ} \mathrm{C}$ for $10 \mathrm{sec}$ and $60^{\circ} \mathrm{C}$ for $15 \mathrm{sec}$. The amplified fluorescent signal was detected by Roche LightCycler 480 (Roche Diagnostics). Relative quantification was assessed using secondary derivative maximum (Roche Diagnostics). Gene expression was normalized to $G A P D H$ and changes in expression measured relative to the control (cells untreated with decitabine). The following PCR primer sequences were used (obtained from Sigma-Genosys, Haverhill, UK): DNMT1 sense: 5'-GAG CCA CAG ATG CTG ACA AA-3'; DNMT1 antisense: 5'-TGC CAT TAA CAC CAC CTT CA-3'; DNMT3a sense: 5'-AGC CCA AGG TCA AGG AGA TT-3'; DNMT3a antisense: 5'-CAG CAG ATG GTG CAG TAG GA-3'; DNMT3b sense: 5'-CCC ATT CGA GTC CTG TCA TT-3'; DNMT3b antisense: 5'-GAT ATT CCC CTC GTG CTT CA-3'; GAPDH sense: 5'-GAG TCA ACG GAT TTG GTC GTA-3'; GAPDH antisense: 5'GCA GAG ATG ATG ACC CTT TTG-3'.

DNA methyltransferase activity. DNA methyltransferase activity of the cells was measured with and without decitabine treatment. Nuclear extracts were prepared according to the manufacturer's protocol (Epigentek, Brooklyn, NY, USA). Cells were treated with $8 \mu \mathrm{M}$ decitabine for $24 \mathrm{~h}$ prior to extraction, and untreated cells were treated with media only. Global DNA methyltransferase activity was measured with the EpiQuik ${ }^{\mathrm{TM}}$ DNA methyltransferase activity/inhibition kit (Epigentek), according to the manufacturer's instructions. Absorbance was measured at $450 \mathrm{~nm}$ (DynaTechMR500, DynaTech Laboratories Inc., Chantilly, VA, USA). DNMT activity/inhibition was calculated in percent relative to the control sample (cells untreated with decitabine), according to the manufacturer's instructions.

Global methylation. Global methylation (Methylamp ${ }^{\text {TM }}$ Global DNA methylation quantification kit, Epigentek) of cells was measured with and without decitabine treatment, according 
to the manufacturer's instructions. Cells were removed from flasks by trypsinisation, washed in PBS, and incubated in $5 \mathrm{ml}$ DNA extraction solution $[0.075 \mathrm{M} \mathrm{NaCl}, 0.024 \mathrm{M}$ EDTA, $\mathrm{pH} 8.0,0.5 \%(\mathrm{w} / \mathrm{v}) \mathrm{SDS}, 1 \mathrm{mg}$ proteinase $\mathrm{K}$ ] overnight and centrifuged with phenol at $12500 \mathrm{x}$ g for $5 \mathrm{~min}$. The resulting upper phase together with $500 \mu 1$ chloroform was centrifuged as above. Again, the upper layer was taken and a total of $50 \mu 13 \mathrm{M}$ sodium acetate (pH 5.2) and $1 \mathrm{ml}$ absolute ethanol was added and inverted several times. For DNA precipitation the tubes were incubated at $-80^{\circ} \mathrm{C}$ for $1 \mathrm{~h}$. DNA was pelleted by centrifugation at $15,000 \mathrm{x}$ g for $10 \mathrm{~min}$ at $4^{\circ} \mathrm{C}$ and washed in $1 \mathrm{ml} 70 \%(\mathrm{v} / \mathrm{v})$ ethanol. The pellets were air-dried and re-suspended in deionised water. Total DNA of $6.6 \mathrm{ng} / \mu 1$ was used per sample. Absorbance was measured at $450 \mathrm{~nm}$ (DynaTech MR500, DynaTech Laboratories) and methylation status was calculated in percent relative to the control sample (cells untreated with decitabine), according to the manufacturer's instructions.

Statistical analysis. Unpaired Student's t-test was performed for all cell viability assays, quantitative PCR, and methylation experiments in order to measure differences between treated and untreated cells or between sensitive and resistant cell lines. All data, unless stated otherwise, are expressed as mean \pm standard error of mean (SEM). A P $<0.05$ was considered statistically significant. SPSS Software, version 17 (SPSS Inc., Chicago, IL, USA) was used for all statistical analyses.

\section{Results}

DNA methylation is altered in docetaxel-resistant breast cancer cells. To examine the contribution of DNA methylation as a mechanism of drug resistance, we compared the global methylation status between docetaxel-sensitive and docetaxelresistant breast cancer cells and also with and without decitabine. The overall methylation status was increased by $82 \pm 9.1 \%$ in MCF-7 docetaxel-resistant cells compared to their parental sensitive line $(\mathrm{P}=0.010)$. In contrast, the methylation status of MDA-MB-231 docetaxel-resistant cells was decreased by $17 \pm 12.8 \%$ but this was not statistically significant. Treatment with decitabine decreased global methylation in both cell lines, however, this was only statistically significant in the MDA-MB-231 cells ( $\mathrm{P}=0.015)$ (Fig. 1). These results suggest that gene expression may be altered due to changes in DNA methylation status in docetaxel-resistant cells.

Docetaxel resistance is associated with decreased DNMT activity. Since global methylation levels differed in MCF-7 and MDA-MB-231 docetaxel-resistant cell lines, we examined the enzymes that catalyse this process using an ELISA-based assay. Levels of DNMT enzyme were measured between docetaxel-sensitive and docetaxel-resistant breast cancer cells and also with and without decitabine. DNMT enzyme activity was decreased by $32 \pm 3.6 \%(\mathrm{P}=0.007)$ and by $33 \pm 1.9 \%$ $(\mathrm{P}=0.035)$ in $\mathrm{MCF}-7$ and MDA-MB-231 resistant cell lines, respectively, compared to their parental sensitive cells. Furthermore, decitabine treatment resulted in a further decrease of DNMT activity in MCF-7 docetaxel-resistant cells $(\mathrm{P}=0.023)$ and MDA-MB-231 docetaxel-resistant cells ( $\mathrm{P}=0.029)$ (Fig. 2). These results suggest that reduced DNA methyltransferase

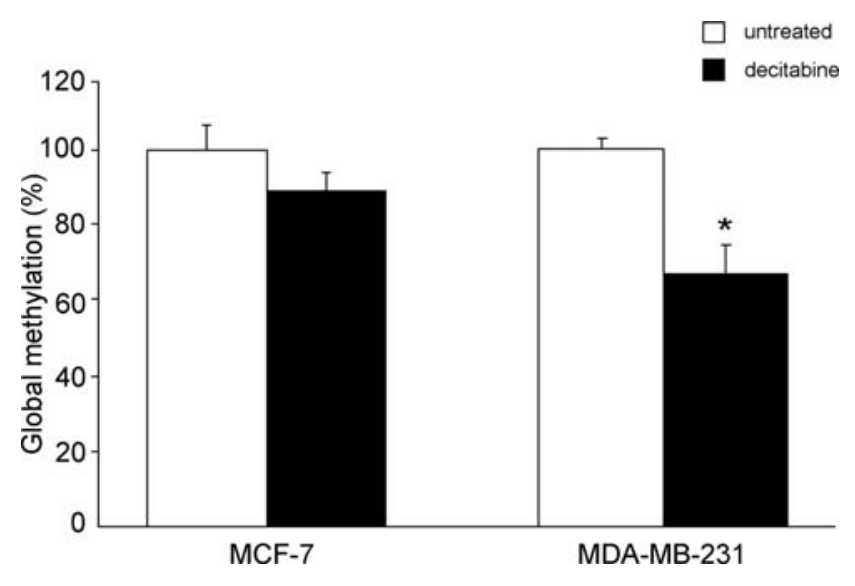

Figure 1. Global methylation in docetaxel-resistant breast cancer cells. Global methylation was measured between MCF-7 and MDA-MB-231 docetaxel-resistant cells and docetaxel-resistant cells treated with $8 \mu \mathrm{M}$ decitabine after $24 \mathrm{~h}$. The results are expressed as a percentage of control ( \pm SEM), which consisted of cells cultured in cell growth media only without decitabine. All experiments were repeated in duplicate with DNA isolated from three independent extractions. ${ }^{*} \mathrm{P}<0.05$.

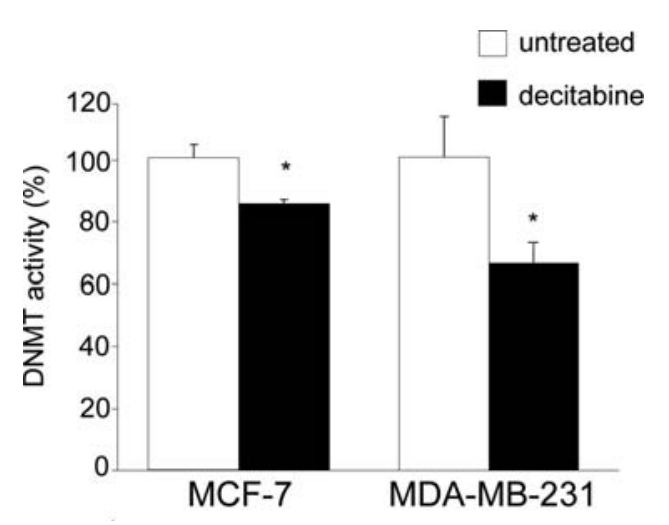

Figure 2. Effect of decitabine on DNA methyltransferase (DNMT) activity. DNMT enzymatic activity was measured between MCF-7 and MDA-MB-231 docetaxel-resistant cells and docetaxel-resistant cells treated with $8 \mu \mathrm{M}$ decitabine after $24 \mathrm{~h}$. The results are expressed as a percentage of control ( \pm SEM), which consisted of cells cultured in cell growth media only without decitabine. All experiments were repeated twice with nuclear DNA isolated from two independent extractions. ${ }^{*} \mathrm{P}<0.05$. Figure with permission from Elsevier (33).

activity, leading to reduced overall DNA methylation, plays a role in acquired resistance to docetaxel in these breast cancer cells.

Altered DNMT gene expression is associated with docetaxel resistance in breast cancer cells. To examine the contribution of different DNA methyltransferases in reducing global DNA methylation, we determined mRNA expression of DNMT1, DNMT3a and DNMT3b using quantitative PCR. In MCF-7 docetaxel-resistant cells, DNMT1 was decreased by $8.4 \pm 11.6 \%$ and $D N M T 3 b$ was decreased by $45.5 \pm 15.4 \%$. In contrast, DNMT3a mRNA expression was increased

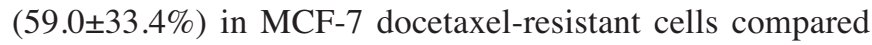
with the parental sensitive cells. None of the DNMT mRNA expression differences in the MCF-7 docetaxel-resistant cells were statistically significant. Expression of DNMT1 was decreased by $31.4 \pm 12.7 \%$ and DNMT3b was decreased by 
$A$

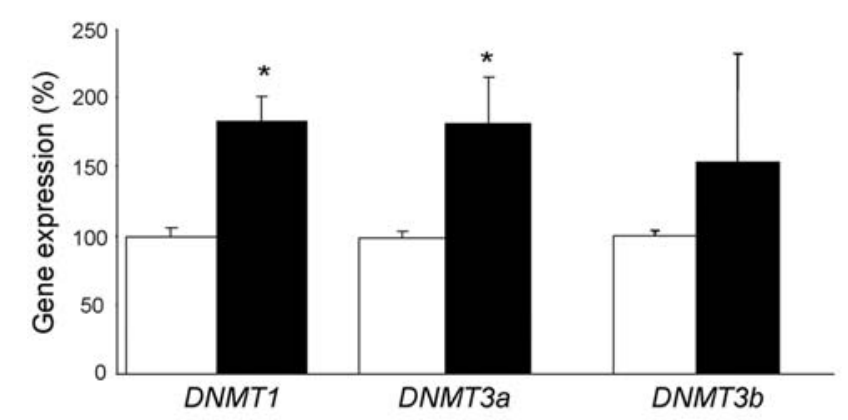

B

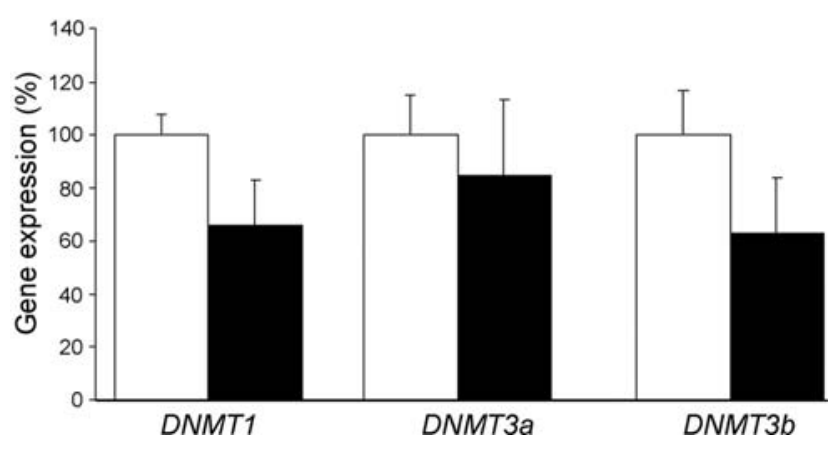

Figure 3. Effect of decitabine on DNA methyltransferase (DNMT) gene expression. Quantitative PCR measured DNMT1, DNMT3a and DNMT3b gene expression between docetaxel-resistant cells and resistant cells treated with $8 \mu \mathrm{M}$ decitabine after $24 \mathrm{~h}$. The results are expressed as a percentage of control $( \pm$ SEM), which consisted of cells cultured in cell growth media only without decitabine. (A) MCF-7 docetaxel-resistant cells. (B) MDA-MB-231 docetaxel-resistant cells. All experiments were repeated in triplicate using two independent cDNA extractions with RNA isolated from three independent RNA extractions. ${ }^{*} \mathrm{P}<0.05$.

$31.7 \pm 14.6 \%$ in MDA-MB-231 docetaxel-resistant breast cancer cells compared with their parental sensitive cells, but did not reach statistical significance. There was no change in the mRNA expression of DNMT3a in MDA-MB-231 docetaxelresistant cells compared with their parental sensitive cells.

Effect of a DNA methylation inhibitor on DNMT gene expression. Docetaxel-resistant breast cancer cells were treated with and without decitabine to examine the effect on DNMT mRNA expression. Decitabine increased DNMT1 $(\mathrm{P}=0.015)$ and DNMT3a $(\mathrm{P}=0.026)$ gene expression in MCF-7 docetaxelresistant cells and also increased $D N M T 3 b$ gene expression but this was not statistically significant (Fig. 3A). In contrast, decitabine decreased the expression of DNMT1, DNMT3a and DNMT3b in MDA-MB-231 docetaxel-resistant cells (Fig. 3B). These results suggest there are differences in DNMT mRNA expression, altered by a DNA methylation inhibitor, between the two docetaxel-resistant breast cancer cell lines, which may reflect differences between the cell types.

Decitabine does not increase sensitivity to docetaxel in breast cancer cells. Since we hypothesised that decitabine would reverse docetaxel resistance, and we identified changes in

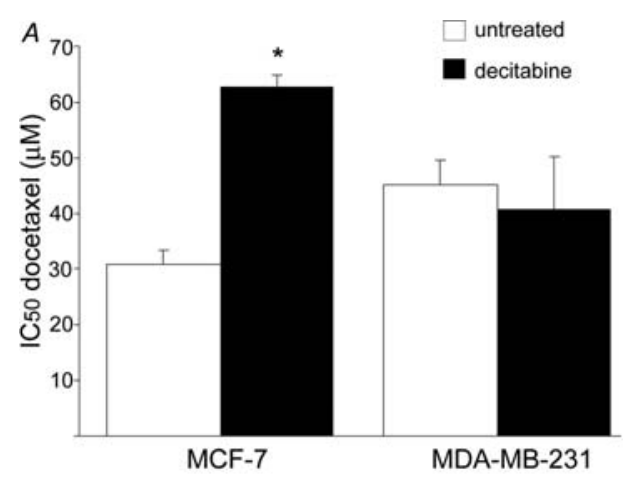

$B$

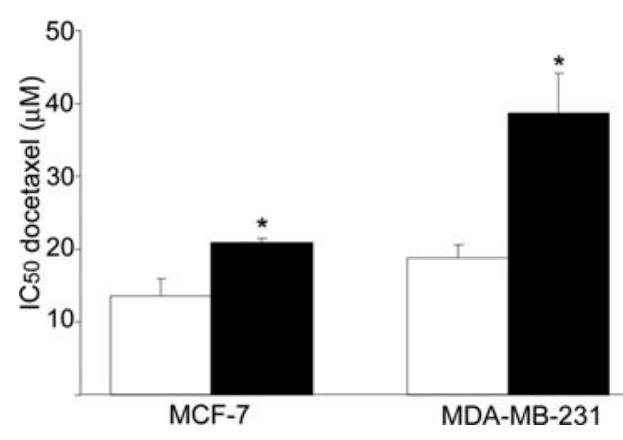

Figure 4. Effect of decitabine and docetaxel on cellular viability. Cellular viability was measured in MCF-7 and MDA-MB-231 breast cancer cells treated with $8 \mu \mathrm{M}$ decitabine after $24 \mathrm{~h}$. The results are expressed as $\mathrm{IC}_{50}$ values $( \pm \mathrm{SD})$ which represent docetaxel concentrations that inhibit $50 \%$ cell growth. (A) Docetaxel-resistant cells. (B) Docetaxel-sensitive cells. All experiments were repeated in triplicate with six replicates per experiment. ${ }^{*} \mathrm{P}<0.05$.

methylation caused by decitabine in our docetaxel-resistant breast cancer cells, we studied the effect of decitabine on response to docetaxel by MTT cell viability assay. Contrary to our hypothesis, we found that $8 \mu \mathrm{M}$ decitabine increased resistance in MCF-7 docetaxel-resistant cells ( $\mathrm{IC}_{50}$-resistant: $30.9 \pm 2.6 \mu \mathrm{M}$ versus $\mathrm{IC}_{50}$-decitabine: $\left.62.7 \pm 2.1 \mu \mathrm{M} ; \mathrm{P}<0.0001\right)$ (Fig. 4A). Other concentrations of decitabine $(0.5,1,2$ and $4 \mu \mathrm{M})$, however, had no statistically significant effect on cell viability of MCF-7 docetaxel-resistant cells. Furthermore, docetaxel response was not altered by any decitabine concentration in MDA-MB-231 docetaxel-resistant cells (Fig. 4A). In addition, we studied the effect of decitabine on parental docetaxel-sensitive cell lines. Decitabine treatment increased resistance to docetaxel in MCF-7 ( $\mathrm{IC}_{50}$-sensitive: 13.6 $\pm 2.4 \mu \mathrm{M}$ versus $\mathrm{IC}_{50}$-decitabine: $20.9 \pm 0.6 \mu \mathrm{M} ; \mathrm{P}=0.007$ ) and MDA-MB-231 ( $\mathrm{IC}_{50}$-sensitive: $18.8 \pm 1.8 \mu \mathrm{M}$ versus $\mathrm{IC}_{50}$-decitabine: $\left.38.6 \pm 5.6 \mu \mathrm{M} ; \mathrm{P}=0.004\right)$ docetaxel-sensitive cell lines (Fig. 4B). Overall these data indicate that treatment with decitabine does not overcome resistance to docetaxel in these specific breast cancer cells.

\section{Discussion}

This is the first study to demonstrate that alterations in the DNA methylation machinery are associated with docetaxel resistance in breast cancer cells. In order to understand mechanisms underlying docetaxel resistance we have 
previously created two in vitro models of acquired docetaxel resistance in human breast cancer using MCF-7 and MDAMB-231 cell lines (12). Global methylation levels were increased in MCF-7 docetaxel-resistant cells but not significantly changed in MDA-MB-231 docetaxel-resistant cells. Decreased DNMT activity, however, and decreased DNMT1 and $D N M T 3 b$ mRNA expression was associated with docetaxel resistance in both cell lines. The DNA methylation inhibitor, decitabine (5-aza-2'-deoxycytidine), as expected, decreased global methylation and DNMT activity but altered the expression of DNMT mRNA differently between the cell lines. Even though docetaxel resistance was associated with changes in the methylation machinery, treatment with a DNA methylation inhibitor, decitabine, did not overcome the resistance to docetaxel in these breast cancer cell lines. Decitabine treatment altered docetaxel response in MCF-7 docetaxel-resistant cells and in the parental MCF-7 and MDA-MB-231 docetaxel-sensitive cell lines, but not in MDA-MB-231 docetaxel-resistant breast cancer cells .

Since the global methylation status in the docetaxel-resistant sublines is altered this suggests a possible association of DNA methylation with drug resistance in these cells. In MCF-7 docetaxel-resistant cells, global methylation was increased by almost 2-fold, which is in agreement with another study that looked at methylation levels in a doxorubicinresistant breast cancer cell line (13). Interestingly, MDAMB-231 docetaxel-resistant cells showed a $40 \%$ decrease in global methylation compared to their parental sensitive cells. Comparison of methylation patterns of specific genes in primary breast tumours showed that tumour-related genes are hypomethylated in estrogen-receptor negative breast tumours (14). In addition, estrogen-receptor expression can be highly influenced by changes in the chromatin structure, which is influenced by epigenetic events driven by DNA methyltransferases or histone deacetylases (15). The differences in global methylation that we observed in MCF-7 and MDA-MB-231 cells may reflect differences between estrogen-receptor positive (MCF-7) and estrogen-receptor negative (MDA-MB-231) cells that have been made resistant to docetaxel. Treatment with decitabine decreased global methylation levels in both docetaxel-resistant sublines, which confirmed that decitabine had a functional effect in our cell lines since it is a global DNA methylation inhibitor.

The identification of differences in global methylation status in the docetaxel-resistant sublines led us to investigate the activity of DNMTs in these cells. DNMT activity was decreased in the MCF-7 docetaxel-resistant cells, which is in contrast to the increased overall methylation status in these cells. DNMT activity was also decreased in MDA-MB-231 docetaxel-resistant cells but this reflected the decreased global methylation in these cells. Decitabine decreased DNMT activity, as expected, in both docetaxel-resistant cell lines. Decreased DNMT activity in cells with increased global methylation was an unexpected finding and suggests there may be other indirect mechanisms by which methylation can be altered in these cells.

To further investigate DNMT activity, we assessed the expression of individual DNMTs using quantitative PCR. In agreement with DNMT enzyme activity, DNMT1 and $D N M T 3 b$ expression were decreased in both docetaxel- resistant cell lines. This is in contrast to other reports associating increased DNMT1 and DNMT3b expression with acquired drug resistance in murine neuroblastoma (16). Analysis of the DNMT3 promoter region revealed that the DNA methylation machinery itself is possibly regulated by DNA methylation $(17,18)$. The 5' upstream region of the $D N M T$ gene showed full promoter activity and this region lies within a CpG-rich site (17). DNMT3a has three promoter regions of which exons $1 \mathrm{~A}$ and $\mathrm{B}$ are flanked by a $\mathrm{CpG}$-rich region (19). In contrast, exon $1 \mathrm{C}$ is flanked by a $\mathrm{CpG}$-poor region. Deletion experiments in this study showed that exon 1A is possibly involved in DNMT3a promoter activity, which suggest that DNA methylation of the promoter region may negatively regulate DNMT activity. DNMT3b, on the other hand, has two promoter regions, exons $1 \mathrm{~A}$ and $\mathrm{B}$. Upstream of exon $1 \mathrm{~A}$ is flanked by a $\mathrm{CpG}$-rich region, whereas exon $1 \mathrm{~B}$ is not. The fact that exons involved in DNMT3 promoter activity are flanked by $\mathrm{CpG}$-rich regions suggests that the DNMT3 promoter, and therefore DNMT3 expression itself, is regulated by DNA methylation, thereby indicating that enzyme activity is regulated by methylation. This may explain why, in the present study, both docetaxelresistant cell lines showed decreased DNMT3b expression and enzyme activity. In addition, it has been shown that different DNMTs are differentially expressed during the cell cycle. DNMT1 and DNMT3b are downregulated in G0/G1 phase whereas DNMT3a shows relatively constant expression throughout the cell cycle in bladder and breast cancer cells (20). This may explain differences in DNMT expression in our present study.

We showed that decitabine treatment decreased the expression of all three DNMT genes in MDA-MB-231 docetaxel-resistant cells. In contrast, however, decitabine treatment increased mRNA expression of DNMT genes in MCF-7 docetaxel-resistant cells. One study examined the expression of DNMT1, DNMT3a and DNMT3b in human colon cancer cells following treatment with another DNA methylation inhibitor, azacytidine (21). This study also demonstrated that a DNA methylation inhibitor reduced expression of DNMT1 and DNMT3a, whereas DNMT3b expression was unaltered. It was not determined, however, if there was a direct interaction between the DNA methylation inhibitor and the DNMT genes. It is not known, therefore, whether azacytidine directly acts on the DNMT genes or indirectly causes inhibition of gene expression by switching on DNMT-silenced genes. There is no evidence in the literature that decitabine, in addition to its binding to DNMT enzymes, can also act on the corresponding DNMT genes. Based on our results we can speculate two possibilities which involve a regulatory feedback mechanism in the DNA methylation machinery (18). Firstly, that decitabine directly acts on the DNMT promoters, which are known to have $\mathrm{CpG}$-rich regions and hence are possibly methylated. Decitabine binding may lead to reduced promoter methylation and, therefore, increased DNMT enzyme production. This feedback loop mechanism may therefore lead to methylation of the promoter regions of the DNMT enzymes themselves, and ultimately result in decreased DNMT gene expression. This, however, was not seen in our docetaxel-resistant cells when treated with decitabine. In contrast, we noted an increased expression 
of DNMT1, DNMT3a and DNMT3b in MCF-7 docetaxelresistant cells treated with decitabine, indicating DNMT gene activation after treatment. This could be due to an indirect interaction of decitabine with the DNMT3 promoter. Addition of decitabine would inhibit DNMT activity, which in turn steadily reduces $D N M T$ promoter methylation and therefore results in transcriptional activation of DNMT1, DNMT3a and $D N M T 3 b$. This possibility may explain the experimental results that we noted in the MCF-7 docetaxel-resistant cells. However, the findings in the MDA-MB-231 cells do not indicate a regulatory feedback mechanism where DNMT mRNA levels match the DNMT activity, and also decitabine consistently inhibits the DNA methylation process in this cell line. Further experiments investigating the effect of decitabine on DNMT promoter methylation would be necessary to define these relationships more clearly. Since treatment with decitabine altered the expression of DNMT1 differently in two docetaxel-resistant breast cancer cell lines, it may suggest there is no preferential binding to DNMT1 or DNMT3. It is unresolved in the literature whether there is a preferential binding of decitabine to DNMT3 (22) or equal binding to DNMT1 and DNMT3 (23).

We identified differences in global methylation, DNMT activity and DNMT gene expression in docetaxel-resistant breast cancer cells, and therefore wanted to test the hypothesis that decitabine could be used to overcome resistance to docetaxel. Previous studies have shown that treatment with decitabine leads to increased sensitivity to chemotherapy drugs such as cisplatin (8) and paclitaxel (24). However, decitabine did not enhance the anti-tumour effect of docetaxel in our docetaxel-resistant breast cancer cells. It was noted also that decitabine actually made both docetaxelsensitive cell lines even less responsive to docetaxel.

There is evidence that decitabine-induced cytotoxicity requires DNMT3a and DNMT3b activity (22). We showed that DNMT3b is decreased in both docetaxel-resistant cell lines, which may also lead to reduced DNMT3 activity, therefore, limit the effect of decitabine-induced cytotoxicity in these cells. There is also evidence that p53 is involved in nucleoside-analogue induced apoptosis (25). Azacytidine treatment in wild-type p53 cells led to G2/M arrest and apoptosis by p53/p21 activation. Knockouts of p53, however, showed diminished apoptotic events due to lack of p53 activity (21). Since MDA-MB-231 cells have a p53 mutant genotype, this may explain why decitabine is unable to exert its cytotoxic effects in these cells. The status of p53 in the cell, however, does not account for lack of response to decitabine in the MCF-7 docetaxel-resistant cells, which have a wild-type p53 genotype. Both of the docetaxel-resistant cell lines used in this study have increased expression of MDRl and increased P-glycoprotein expression (26). MDRl promoter hypomethylation has been associated with drug resistance in acute myeloid leukaemia (27) and also in human breast cancer (28). Addition of a DNA methylation inhibitor, such as decitabine, could increase the state of hypomethylation and therefore stabilise the expression of MDRl. This possibility may explain why resistance to docetaxel was increased in our cells after addition of decitabine. In addition, epigenetic regulation like DNA methylation was associated with abnormal expression of class III B-tubulin mRNA and protein expression in ovarian cancer cells (29). Class III B-tubulin expression was increased following decitabine treatment, but only in ovarian cancer cell lines that initially had low levels of class III ß-tubulin. This implies that class III ß-tubulin undergoes methylation and can be increased by decitabine. We have previously shown that docetaxel resistance is associated with increased expression of class III B-tubulin in MCF-7 breast cancer cells (3). This suggests an association of DNA methylation and B-tubulin expression in our MCF-7 cell model, whereby decitabine increases class III B-tubulin levels and therefore may decrease response to docetaxel.

In conclusion, we have shown docetaxel resistance to be associated with differences of DNA methylation status and DNA methyltransferase enzyme expression. Treatment with decitabine altered the methylation machinery in these cells; however, docetaxel resistance could not be reversed using decitabine as a DNA methylation inhibitor. It has been shown that decitabine on its own does not exert its antitumour effects (30) but drug resistance may be overcome in combination with histone deacetylation inhibitors $(31,32)$. Our study suggests that decitabine may exert its effects in a cell line specific manner, which may have possible clinical implications if using this inhibitor for the treatment of breast cancer. The use of epigenetic therapies, as a strategy to overcome drug resistance, needs to be investigated more fully to determine their usefulness in different cancers and for different chemotherapy drugs.

\section{Acknowledgements}

The authors especially thank Dr Alun Hughes for his help and support with performing and analysing the quantitative PCR studies. This study was supported by a Dr James Alexander Mearns studentship and the Fraserburgh Moonlight Prowl Fund.

\section{References}

1. Jones SE, Erban J, Overmoyer B, et al: Randomized phase III study of docetaxel compared with paclitaxel in metastatic breast cancer. J Clin Oncol 23: 5542-5551, 2005.

2. Smith IC, Heys SD, Hutcheon AW, et al: Neoadjuvant chemotherapy in breast cancer: significantly enhanced response with docetaxel. J Clin Oncol 20: 1456-1466, 2002.

3. Shalli K, Brown I, Heys SD and Schofield AC: Alterations of beta-tubulin isotypes in breast cancer cells resistant to docetaxel. FASEB J 19: 1299-1301, 2005.

4. Noguchi S: Predictive factors for response to docetaxel in human breast cancers. Cancer Sci 97: 813-820, 2006.

5. Miyoshi Y, Taguchi T, Kim SJ, Tamaki Y and Noguchi S: Prediction of response to docetaxel by immunohistochemical analysis of CYP3A4 expression in human breast cancers. Breast Cancer 12: 11-15, 2005.

6. Modok S, Mellor HR and Callaghan R: Modulation of multidrug resistance efflux pump activity to overcome chemoresistance in cancer. Curr Opin Pharmacol 6: 350-354, 2006.

7. Staub J, Chien J, Pan Y, et al: Epigenetic silencing of HSulf-1 in ovarian cancer: implications in chemoresistance. Oncogene 26: 4969-4978, 2007.

8. Zhang XT, Yashiro M, Ren J and Hirakawa K: Histone deacetylase inhibitor, trichostatin A, increases the chemosensitivity of anticancer drugs in gastric cancer cell lines. Oncol Rep 16: 563-568, 2006.

9. Plumb JA, Strathdee G, Sludden J, Kaye SB and Brown R: Reversal of drug resistance in human tumor xenografts by 2'deoxy-5-azacytidine-induced demethylation of the hMLH1 gene promoter. Cancer Res 60: 6039-6044, 2000. 
10. Fulda S, Küfer MU, Meyer E, van Valen F, DockhornDworniczak B and Debatin K: Sensitization of resistant tumor cells for chemotherapy- or death-receptor-induced, apoptosis by restoration of absent caspase-8 expression through demethylation or caspase-8 gene transfer. Oncogene 20: 5865-5877, 2001.

11. Shang D, Ito N, Kamoto T and Ogawa O: Demethylating agent 5-aza-2'-deoxycytidine enhances susceptibility of renal cell carcinoma to paclitaxel. Urology 69: 1007-1012, 2007.

12. Brown I, Shalli K, McDonald SL, et al: Reduced expression of p27 is a novel mechanism of docetaxel resistance in breast cancer cells. Breast Cancer Res 6: 601-607, 2004.

13. Segura-Pacheco B, Perez-Cardenas E, Taja-Chayeb L, et al: Global DNA hypermethylation-associated cancer chemotherapy resistance and its reversion with the demethylating agent hydralazine. J Transl Med 4: 32, 2006.

14. Sunami E, Shinozaki M, Sim MS, et al: Estrogen receptor and HER2/neu status affect epigenetic differences of tumor-related genes in primary breast tumors. Breast Cancer Res 10: R46, 2008.

15. Feng W, Shen L, Wen S, et al: Correlation between CpG methylation profiles and hormone receptor status in breast cancers. Breast Cancer Res 9: R57, 2007.

16. Qui YY, Mirkin BL and Dwidevi RS: Differential expression of DNA-methyltransferases in drug resistant murine neuroblastoma cells. Cancer Detect Prev 26: 444-453, 2002.

17. Ishida C, Ura K, Hirao A, et al: Genomic organization and promoter analysis of the DNMT3b gene. Gene 310: 151-159, 2003.

18. Slack A, Cervoni N, Pinard M and Szyf M: Feedback regulation of DNA methyltransferase gene expression by methylation. Eur J Biochem 264: 191-199, 1999

19. Yanagisawa $\mathrm{Y}$, Ito E, Yuasa $\mathrm{Y}$ and Maruyama K: The human DNA methyltransferases DNMT3A and DNMT3B have two types of promoters with different $\mathrm{CpG}$ contents. Biochim Biophys Acta 1577: 457-465, 2002.

20. Robertson KD, Keyomarsi K, Gonzales FA, Velicescu M and Jones PA: Differential mRNA expression of the human DNA methyltransferases (DNMTs) $1,3 a$ and $3 b$ during the G0/G1 and $\mathrm{S}$ phase transition in normal and tumor cells. Nucleic Acids Res 28: 2108-2113, 2000

21. Schneider-Stock R and Ocker M: Epigenetic therapy in cancer: molecular background and clinical development of histone deacetylase and DNA methyltransferase inhibitors. I. Drugs 10: $557-561,2007$.
22. Oka M, Meacham AM, Hamazaki T, Rodic N, Chang LJ and Terada N: De novo DNA methyltransferases DNMT3a and DNMT3b primarily mediate the cytotoxic effect of 5-aza-2'deoxycytidine. Oncogene 24: 3091-3099, 2005.

23. Weisenberger DJ, Velicescu M, Cheng JC, Gonzales FA, Liang G and Jones PA: Role of the DNA methyltransferase variant DNMT3b3 in DNA methylation. Mol Cancer Res 2: 62-72, 2004.

24. Yanokura M, Banno K, Kawaguchi M, et al: Relationship of aberrant DNA hypermethylation of CHFR with sensitivity to taxanes in endometrial cancer. Oncol Rep 17: 41-48, 2007.

25. Pulukuri SM and Rao JS: Activation of $\mathrm{p} 53 / \mathrm{p} 21^{\text {Wafl/Cip } 1}$ pathway by 5 -aza-2'-deoxycytidine inhibits cell proliferation, induces pro-apoptotic genes and mitogen-activated protein kinases in human prostate cancer cells. Int J Oncol 26: 863-871, 2005.

26. McDonald SL, Stevenson DA, Moir SE, et al: Genomic changes identified by comparative genomic hybridisation in docetaxelresistant breast cancer cell lines. Eur J Cancer 41: 1086-1094, 2005.

27. Nakayama M, Wada M, Harada T, et al: Hypomethylation status of $\mathrm{CpG}$ sites at the promoter region and overexpression of the human MDR1 gene in acute myeloid leukemias. Blood 92: 4296-4307, 1998 .

28. Goffin J and Eisenhauer E: DNA methyltransferase inhibitorsstate of the art. Ann Oncol 13: 1699-1716, 2002.

29. Izutsu N, Maesawa C, Shibazaki M, et al: Epigenetic modification is involved in aberrant expression of class III B-tubulin, TUBB3, in ovarian cancer cells. Int J Oncol 32: 1227-1235, 2008.

30. Chekhun VF, Kulik GI, Yurchenko OV, et al: Role of DNA hypomethylation in the development of the resistance to doxorubicin in human MCF-7 breast adenocarcinoma cells. Cancer Lett 231: 87-93, 2006.

31. Hostetter CL, Licata LA and Keen JC: Timing is everything: order of administration of 5-aza 2' deoxycytidine, trichostatin A and tamoxifen changes estrogen receptor mRNA expression and cell sensitivity. Cancer Lett 275: 178-184, 2009.

32. Steele N, Finn P and Plumb JA: Combined inhibition of DNA methylation and histone acetylation enhances gene re-expression and drug sensitivity in vivo. Br J Cancer 100: 758-763, 2009.

33. Kastl L, Brown I and Schofield AC: Effects of decitabine on the expression of selected endogenous control genes in human breast cancer cells. Mol Cell Probes (In press). 